\title{
Gender and individual differences in cueing effects: Visuospatial attention and object likability
}

\author{
Takashi Mitsuda $^{1} \cdot$ Mio Otani $^{1} \cdot$ Sayana Sugimoto ${ }^{1}$
}

Published online: 3 May 2019

(C) The Psychonomic Society, Inc. 2019

\begin{abstract}
An arrow marker appearing in a field of view shifts the observer's attention in its indicated direction. On the other hand, the gaze of another individual not only shifts the observer's attention but also increases the likability of objects in the direction of their gaze. These effects occur even when the arrow markers or gaze cues are presented for very short periods so that the observer is unaware of them. To expand on the findings of previous studies, we conducted two experiments. In the first we examined the changes in likability and attentional shift induced by supraliminal and subliminal arrow markers, using 40 male and 40 female participants. We found, contrary to previous findings, that supraliminal arrow markers not only shifted the observers' attention but slightly increased object likability. On the other hand, subliminal arrow markers only shifted the observers' attention. The attentional shift was stronger for female than for male participants in the case of supraliminal, but not of subliminal, markers. The second experiment verified the changes in likability induced by supraliminal arrow markers and compared the effects with those of supraliminal gaze cues among 40 female participants. We found that the liking effect was observed for both arrow markers and gaze cues. However, both effects were small, and no significant difference was found between them. Overall, these results show that gender and individual differences have considerable impacts on cueing effects.
\end{abstract}

Keywords Attention $\cdot$ Preference $\cdot$ Gender differences $\cdot$ Cueing $\cdot$ Unconsciousness

The human visual system can direct humans' attention toward a part of the information projected on their retinas, regardless of their conscious awareness of that information. When a visual stimulus such as an arrow appears in the visual field, people automatically shift their attention toward the direction indicated by the stimulus. This automatic process has been verified by the reduction of reaction times to a visual target in the direction indicated by a visual stimulus as compared to one in the opposite direction (Posner, 1980).

Visual stimuli that direct the observer's attention include symbolic markers such as arrows, letters, and gaze. The gaze of another individual not only shifts the observer's attention, but also increases the likability of the objects that are gazed at (e.g., Bayliss, Paul, Cannon, \& Tipper, 2006; van der Weiden, Veling, \& Aarts, 2010). On the other hand, neither an arrow marker

Takashi Mitsuda

mitsuda@is.ritsumei.ac.jp

1 College of Information Science and Engineering, Ritsumeikan University, Kusatsu, Shiga, Japan
(Bayliss et al., 2006) nor a pointing hand (Ulloa, Marchetti, Taffou, \& George, 2015) increases the likability of objects pointed toward by these stimuli. Therefore, gaze is thought to be processed by a higher cognitive mechanism. The facial expression (Bayliss, Frischen, Fenske, \& Tipper, 2007; Bayliss, Schuch, \& Tipper, 2010; Becker, 2010; Canadas \& Mast, 2017; Niedzwiecka \& Tomalski, 2015; Ponari, Trojano, Grossi, \& Conson, 2013), trustworthiness (King, Rowe, \& Leonards, 2011; Süßenbach \& Schönbrodt, 2014), and dominance (Jones et al., 2010) of a gaze sender modulates the cueing effects, which also demonstrates the involvement of a higher cognitive mechanism in processing gaze cues.

Recently, Mitsuda and Masaki (2018) showed that even when gaze cues were presented for a short period of time and the observers remained unaware of them, the gaze cues shifted the spatial attention of the observers and increased the likability of the objects in the direction indicated by the gaze. This shows that the modulation of object likability by gaze cueing, which was thought to be processed by a higher cognitive mechanism, is also triggered by an automatic, unconscious process. On the other hand, previous studies have shown that symbolic markers also shift an observers' attention when they are presented 
subliminally (Gabay, Avni, \& Henik, 2012; Gayet, Van der Stigchel, \& Paffen, 2014; Reuss, Pohl, Kiesel, \& Kunde, 2011; Sato, Okada, \& Toichi, 2007; Serrien, Sovijärvi-Spapé, \& Rana, 2012; Xu, Zhang, \& Geng, 2011). However, no articles have reported whether subliminal symbolic markers increase the likability of the objects they indicate. According to a study reporting that supraliminal symbolic markers did not modulate the likability of objects (Bayliss et al., 2006), subliminal symbolic markers may also not modulate likability. However, no other articles have verified the modulation of object likability by supraliminal symbolic markers.

Previous studies have shown that repetitive observations of an object increase the likability of the object; this is known as the "mere-exposure effect" (Bornstein, 1989; Zajonc, 1968). One of the hypotheses concerning its origin is the misattribution of fluency. Repetitive exposure to an object eases the process of perceiving the object, and the resultant perceptual fluency is misattributed to the likability of the object. According to this hypothesis, if perceiving an object at the location attended to is easier than perceiving another object at another location, the difference in perceptual fluency may cause a difference in the likability of these objects. Therefore, this study hypothesized that the spatial attention caused by symbolic markers increases the likability of objects at the indicated location. According to this hypothesis, symbolic markers, which shift the observer's spatial attention, must increase the likability of the object they indicate, regardless of whether they are supraliminal or subliminal. Consequently, in the present study we examined likability modulation by supraliminal and subliminal arrow markers.

At the same time, in the present study we also aimed to examine gender differences in the modulation of spatial attention and object likability by symbolic markers. Previous studies have shown that female observers shifted their spatial attention under supraliminal visual stimuli more strongly than did male observers (Alwall, Johansson, \& Hansen, 2010; Bayliss, di Pellegrino, \& Tipper, 2005; Cooney, Brady, \& Ryan, 2017; Deaner, Shepherd, \& Platt, 2007; Feng et al., 2011; Liu, Hu, Fan, \& Wang, 2013; Merritt et al., 2007). On the other hand, no study has reported a gender difference in spatial attention under subliminal visual markers, except for one study using sexual pictures (Gillath, Mikulincer, Birnbaum, \& Shaver, 2007). Furthermore, regardless of whether the stimuli were supraliminal or subliminal, gender differences in the modulation of object likability by gaze cueing or symbolic markers have not been reported by previous studies. The one study showing an increase of likability induced by a subliminal gaze cue reported that female observers were affected by the gaze cue more than were male observers, but that the difference between genders was not statistically significant (Mitsuda \& Masaki, 2018). Furthermore, a study that reported no modulation of object likability by arrow cues (Bayliss et al., 2006) employed an imbalanced male-female ratio of participants (three male and 21 female). Accordingly, the examination of gender differences is fundamental to determining whether symbolic markers modulate object likability. The present study consequently included more participants than previous studies had, in order to investigate such a difference.

In the present study, we first examined the attentional shifts induced by arrow markers in terms of gender and stimulus condition (i.e., whether a stimulus was supraliminal or subliminal). Next, in this study we examined the effects of gender and stimulus condition on the modulation of object likability by arrow markers. Finally, we compared the magnitude of the liking effect induced by arrow markers with that induced by gaze cues.

\section{Experiment 1}

In this experiment, we examined attentional shifts induced by arrow markers in terms of gender and stimulus condition (i.e., whether a stimulus was supraliminal or subliminal). Next, we examined the effects of gender and stimulus condition on the modulation of object likability by arrow markers.

\section{Method}

\section{Participants}

Eighty students from a Japanese university ( 40 women and 40 men; $M_{\text {age }}=21.2$ years, $S D=1.6$ ) participated in all tasks. We obtained informed consent from all participants, and they received $1,000 ¥$ for their participation. This experiment was approved by the Ritsumeikan University ethics review committee for research involving human participants and was conducted in accordance with the Declaration of Helsinki.

\section{Apparatus}

The stimuli were presented on a 24-in. display monitor (XL2411Z; BenQ Corp., Taipei, Taiwan) at $120 \mathrm{~Hz}$, using a homemade program written in the $\mathrm{C}$ programming language.

\section{Stimuli}

The stimuli consisted of a bar and two arrows, one arrow pointing right and the other left, in black on a white background (H $8.4 \times$ V $8.4 \mathrm{deg}$ ), that could be displayed as needed. The bar measured $0.1 \times 1.5 \mathrm{~cm}$, and the arrows consisted of a bar and two arrowheads $(0.7 \times 0.7 \mathrm{~cm}$; see Fig. 1$)$.

For the likability evaluation task, we used nonsensical geometrical figures created by Endo, Saiki, Nakao, and Saito (2003). All the figures were composed of hand-drawn contours in which the lines did not cross each other. We scanned 


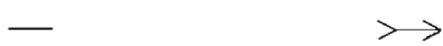

Bar

Arrows

Fig. 1 The bar and arrows used in the present experiments to indicate an item presented on one side of the screen

100 figures from the article and changed their sizes to be equal to that of the arrow images. Ten university students, who did not take part in the main experiment, were recruited for the purpose of selecting and testing the images. These ten students ( $M_{\text {age }}=20.7$ years, $S D=1.5$ ) rated their preference for each figure on a 7-point scale, from 1 (not favorable at all) to 7 (very favorable). We then used 80 figures (preference rating $M$ $=4.00, S D=0.32$ ) selected from the 100 figures of the initial sample, by eliminating the ten highest- and ten lowest-rated figures. These nonsensical geometric figures were the same as those used in Mitsuda and Masaki (2018), which had examined changes in likability induced by subliminal gaze cues.

\section{Procedure}

All participants carried out a target detection task (Task 1) followed by a likability evaluation task (Task 2). Each task consisted of 160 trials using supraliminal or subliminal arrow markers. The participants performed the supraliminal trials ( 80 trials) and subliminal trials (80 trials) serially in each task. The order of the supraliminal and subliminal trials was counterbalanced across participants.

After completing the two tasks, all participants were informed about the subliminal arrow stimuli and completed a survey asking whether they had noticed the subliminal presentation of arrow markers during the two tasks. They then undertook an arrow detection task (Task 3), in which the subliminal arrow markers were presented as they had been in the target detection task, before answering the same survey again.

Task 1: The target detection task Figures $2 \mathrm{a}$ and $2 \mathrm{~b}$ present the time courses of the supraliminal and subliminal trials, respectively. A white fixation cross (H $1.6 \times$ V $1.6 \mathrm{deg})$ appeared for $1,000 \mathrm{~ms}$ at the center of the screen, followed by the image of the bar. After 1,000 ms, the bar changed to an arrow that pointed either right or left. In the supraliminal trials, the arrow was presented for $1,000 \mathrm{~ms}$ followed by a circular target (1.6 deg in diameter) on the right or left side of the screen. Participants had to report the position of the target as soon as possible by pressing the Z key (left) or the X key (right). In the subliminal trials, the arrow appeared for $8 \mathrm{~ms}$ followed by mask images of the same size as the arrow image for 192 $\mathrm{ms}$, and then by the same arrow for another $8 \mathrm{~ms}$. This sequence was repeated. The same arrow image appeared five times interspersed with the mask images, for a total duration of 1,000 ms. After the mask images had disappeared, a circular target appeared, and participants had to report the position of the target, as in the supraliminal trials.
During the subliminal arrow presentations for $1,000 \mathrm{~ms}$, the mask images were updated every $8 \mathrm{~ms}$. Each mask image was composed of 5,000 white dots $(\mathrm{H} 0.1 \times \mathrm{V} 0.1 \mathrm{deg})$ located at a randomized position on a black background. After participants had reported the position of the circular target, the central fixation cross appeared for the next trial. Participants performed 160 trials, in which the arrow pointed either right or left ( 80 trials each). The arrow pointed at the circular target in 80 trials, and pointed in the direction opposite the circular target in 80 trials. The circular target appeared randomly on the right or left side of the screen equal numbers of times. Participants practiced the trials until they had become used to the task before data collection was started.

Task 2: The likability evaluation task Figures $2 \mathrm{c}$ and $2 \mathrm{~d}$ present the time courses of the stimulus presentations for the supraliminal and subliminal trials, respectively. The presentations of supraliminal and subliminal arrows were the same as in Task 1. In this task, however, a nonsensical image appeared to the right or left of the arrow and mask images. Participants then evaluated the likability of the nonsensical image on a 7-point scale, ranging from - 3 (not favorable at all) to +3 (very favorable), by clicking an image of the number on the screen after the mask image had disappeared. The nonsensical image remained present until participants had answered. The arrow pointed toward the nonsensical figures (indicated targets) or in the opposite direction (averted targets) equal numbers of times. The positions of the nonsensical figures and the directions of the arrows were counterbalanced. The combinations of indicated and averted targets were also counterbalanced. The nonsensical figures were presented in a random order. Each of the 80 nonsensical figures appeared only once in both the supraliminal and subliminal trials.

Task 3: The arrow detection task After performing Tasks 1 and 2 , participants completed a survey about whether they had noticed that subliminal arrows had been interspersed with the mask images. After the survey, participants were informed that subliminal arrows had been presented. Then they performed an arrow detection task. The subliminal arrows were presented in the same fashion as in the target detection task (Task 1). Participants had to report the arrow direction by pressing the $\mathrm{Z}$ key (left) or the $\mathrm{X}$ key (right) after the disappearance of the mask images. Even when participants did not detect the arrow direction, they had to report a direction intuitively. They performed 40 trials, in which the arrow direction was to either the right (20 trials) or the left (20 trials); the arrow direction was determined in a random order. After the data collection, participants were asked whether they had been able to detect the arrow direction. The experiment took about 60 min. 

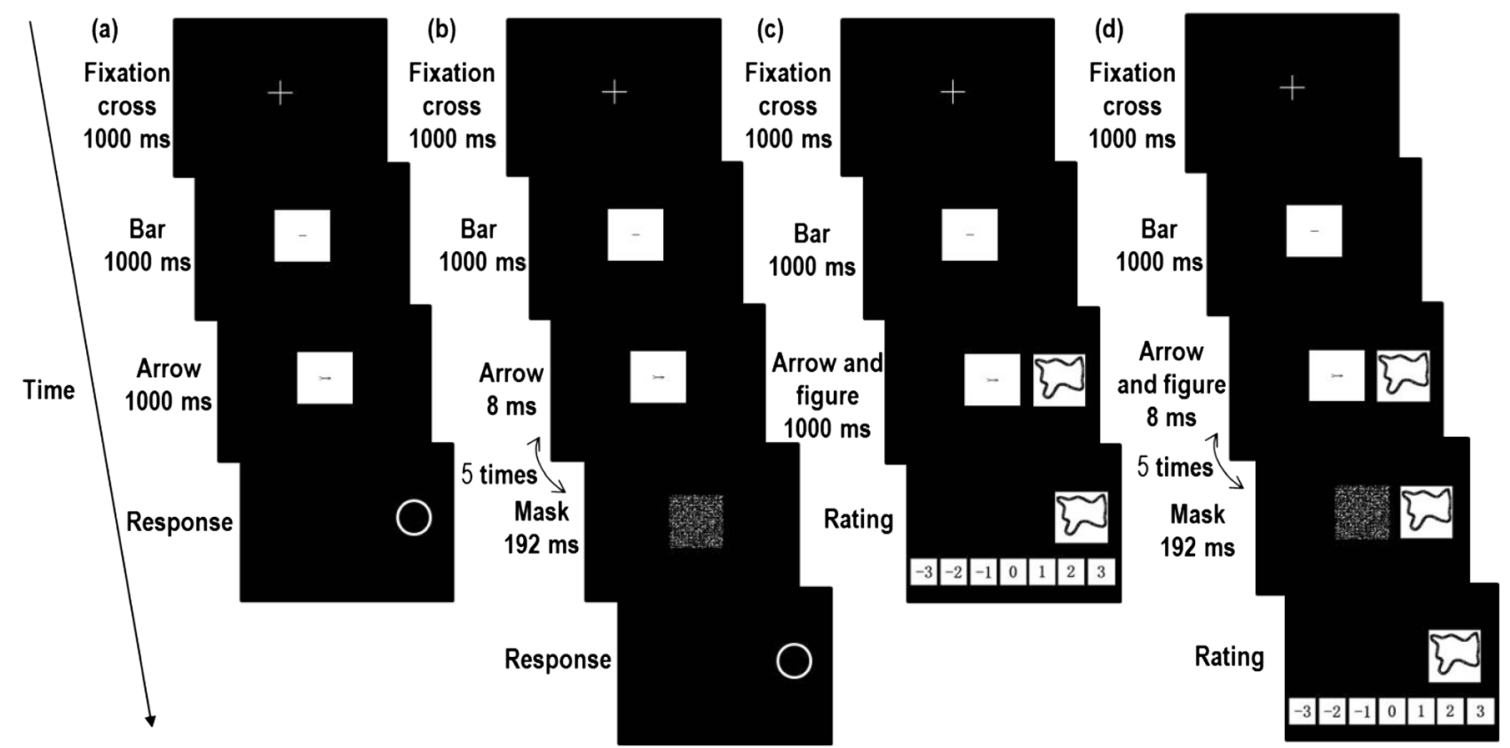

Fig. 2 Illustration of the time courses of the target detection task with (a) supraliminal arrows and (b) subliminal arrows (Task 1), and of the likability evaluation task with (c) supraliminal arrows and (d) subliminal arrows (Task 2)

\section{Results}

During inquiries after the recordings, one of the male participants reported that he was able to detect the arrow direction even when it was presented subliminally for a short period. In accordance with his report, his correct answer rate for the arrow direction in the arrow detection task (Task 3) was .925. This rate was significantly higher than chance (binomial test, $p<.0001)$. On the other hand, one female participant reported that she was not able to detect the arrow direction; however, her correct answer rate was .825 , which was also significantly higher than chance (binomial test, $p<.0001$ ). Excluding these individuals, the highest and lowest correct answer rates among the rest of the participants were .65 (binomial test, $p=.005$ ) and .3875 (binomial test, $p=.0283$ ), respectively, which were not significant when the significance level was adjusted for the number of participants ( 80 participants). Consequently, we analyzed the behaviors of 78 participants. The correct answer rate for these participants $(M=$ .508 ) did not differ significantly from chance, $t(77)=1.60, p$ $=.11$. There was no significant difference in the correct answer rates between genders, $t(76)=0.55, p=.58$ (male: $M=$ .505 , female: $M=.511$ ).

For the target detection task (Task 1), a two-way repeated measures analysis of variance (ANOVA) of target position (cued or uncued) and stimulus condition (supraliminal or subliminal) showed a significant main effect of the target position, $F(1,77)=27.70, p<.001, \eta_{\mathrm{p}}{ }^{2}=.27$, and a significant interaction between these variables, $F(1,77)=6.21, p=.015, \eta_{\mathrm{p}}{ }^{2}=$ .08 . The main effect of stimulus condition was not significant, $F(1,77)=0.70, p=.41$. The reaction time to targets that appeared in the direction indicated by a supraliminal arrow $(M=372 \mathrm{~ms}, S E M=9 \mathrm{~ms})$ was significantly shorter than that to targets that appeared in the opposite direction $(M=385 \mathrm{~ms}$, $S E M=10 \mathrm{~ms}$ ) in a paired-sample $t$ test, $t(77)=4.82, p<$ $\left..0001, d_{z}=0.55\right)$. Even when arrow markers were presented for a short period, the reaction time to the indicated targets $(M$ $=383 \mathrm{~ms}, S E M=13 \mathrm{~ms}$ ) was significantly shorter than that to the averted targets $(M=389 \mathrm{~ms}, S E M=13 \mathrm{~ms}), t(77)=2.91, p$ $=.0048, d_{z}=0.33$. The cueing effect (i.e., the reaction time to averted targets minus the reaction time to indicated targets) was significantly greater when arrow markers were supraliminal $(M=13 \mathrm{~ms})$ than when they were subliminal $(6 \mathrm{~ms}), t(77)$ $=2.49, p=.015, d_{z}=0.28$.

Figure 3 shows histograms of the cueing effects by observer gender and stimulus condition (supraliminal or subliminal). A two-way repeated measures ANOVA showed a significant main effect of stimulus condition, $F(1,76)=6.56, p=.012$, $\eta_{\mathrm{p}}{ }^{2}=.08$, and a significant interaction between stimulus condition and gender, $F(1,76)=5.36, p=.023, \eta_{\mathrm{p}}{ }^{2}=.07$. When the arrow markers were supraliminal, the cueing effect was significant for female participants, $t(38)=5.31, p<.0001, d_{z}=$ 0.85 ( $M=20 \mathrm{~ms}, S E M=4 \mathrm{~ms})$, but not for male participants, $t(38)=1.76, p=.087, d_{z}=0.28(M=7 \mathrm{~ms}, S E M=4 \mathrm{~ms})$. There was a significant difference in the cueing effect between genders, $t(76)=2.47, p=.016, d=0.57$. When supraliminal arrow markers were presented, 35 of the 39 female participants $(90 \%)$ responded faster to the indicated targets than to the averted ones. On the other hand, only 20 of the 39 male participants $(51 \%)$ responded faster to the indicated targets. When the arrow markers were presented subliminally, the cueing effect was significant for male participants, $t(38)=$ $2.27, p=.029, d_{z}=0.36(M=6 \mathrm{~ms}, S E M=3 \mathrm{~ms})$, but not for female participants, $t(38)=1.83, p=.075, d_{z}=0.29(M=5$ $\mathrm{ms}, S E M=3 \mathrm{~ms})$. However, there was no significant difference between genders: $t(76)=0.22, p=.83$. When the arrow 
(a)

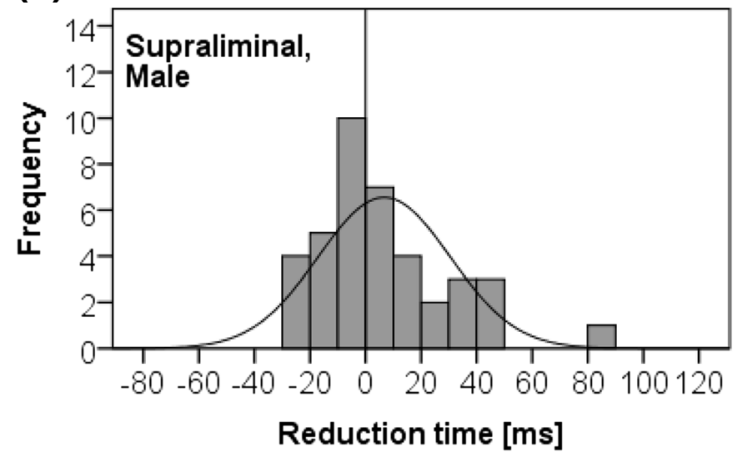

(c)

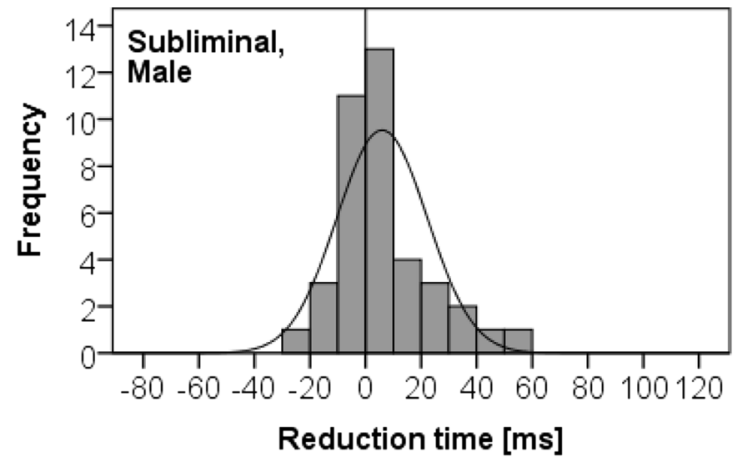

(b)

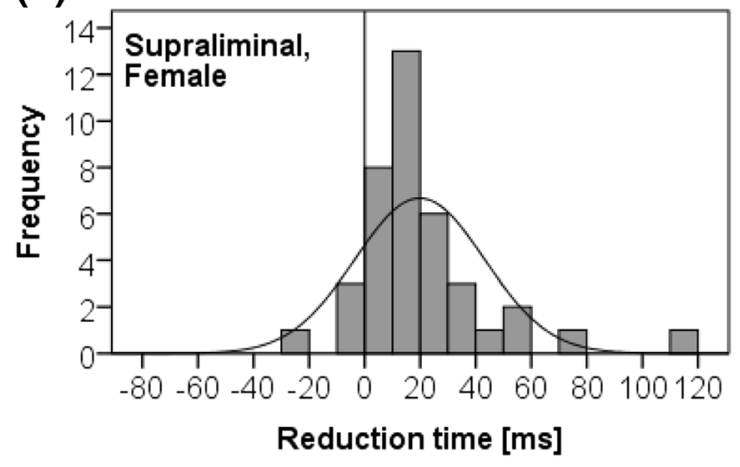

(d)

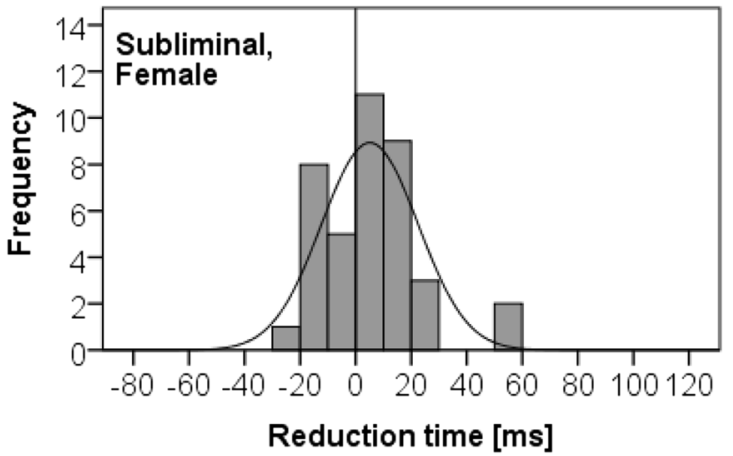

Fig. 3 Histograms showing cueing effects in reaction times (i.e., reaction time to an averted target minus reaction time to an indicated target) by gender of the observer and stimulus condition (supraliminal or subliminal). Bell-shaped curves represent the best-fit normal distributions

markers were presented subliminally, the numbers of participants who responded faster to the indicated targets were almost equal for both genders: 24 (62\%) for male participants and $25(64 \%)$ for female participants.

Turning to the results of the likability evaluation task (Task 2), a two-way repeated measures ANOVA of target position and stimulus condition (supraliminal or subliminal) showed a significant interaction, $F(1,77)=4.30, p=.041, \eta_{\mathrm{p}}{ }^{2}=.05$, but no significant main effect was observed for either target position, $F(1,77)=2.61, p=.11$, or stimulus condition, $F(1,77)=$ $0.62, p=.43)$. When supraliminal arrow markers were presented, the likability of indicated targets $(M=-0.174)$ was significantly higher than that of averted ones $(M=-0.279), t(77)=$ $2.21, p=.030, d_{z}=0.25$. When the arrow markers were presented subliminally, however, there was no significant difference in target evaluations between the target positions, $t(77)=$ $0.82, p=.22$ (cued: $M=-0.255$, uncued: $M=-0.263$ ).

Figure 4 shows histograms of the cueing effects on target evaluation (i.e., the average rating of averted targets minus the average rating of indicated targets) by observer gender and stimulus condition. A two-way repeated measures ANOVA of stimulus condition and gender showed only a significant main effect of stimulus condition, $F(1,76)=4.30, p=.042$, $\eta_{\mathrm{p}}{ }^{2}=.05$. Neither the main effect of gender, $F(1,76)=0.23, p$ $=.64$, nor the interaction, $F(1,76)=0.96, p=.33$, was significant. Among the female participants, when supraliminal arrow markers were presented, evaluations of the indicated targets $(M=-0.246)$ were significantly higher than those of the averted targets $(M=-0.390), t(38)=2.06, p=.046, d_{z}=$ 0.33. On the other hand, among the male participants, the evaluations did not differ according to target position, $t(38)$ $=1.02, p=.32, d_{z}=0.16$ (cued: $M=-0.103$, uncued: $M=-$ $0.167)$. When the arrow markers were presented subliminally, the target evaluations did not differ according to target position in participants of either gender [male: $t(38)=0.27, p=$ .79; female: $t(38)=0.04, p=.97]$. As is shown in Fig. 4 , the cueing effect for supraliminal stimuli (1.9) was much larger for one female participant than for the others. When this participant was excluded from the analysis, among the female participants, the evaluation of the targets pointed to by supraliminal arrows $(M=-0.293)$ became only slightly higher than that of the other targets $(M=-0.390), t(37)=1.82, p=.077$, $d_{z}=0.30$.

Finally, we performed correlation analyses to assess the relationship between the effects of cueing on reaction times and target evaluations for each task and stimulus condition (supraliminal or subliminal). The cueing effect on reaction times in the target detection task (Task 1) did not correlate significantly with that on target evaluations in the likability evaluation task (Task 2) for any combinations of stimulus condition and gender $(r s<.083, p s>.62)$. The cueing effect on reaction times for supraliminal arrow markers did not 
(a)

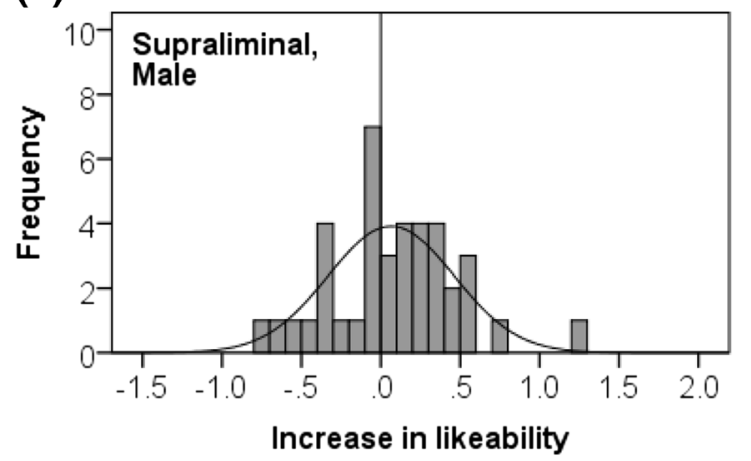

(c)

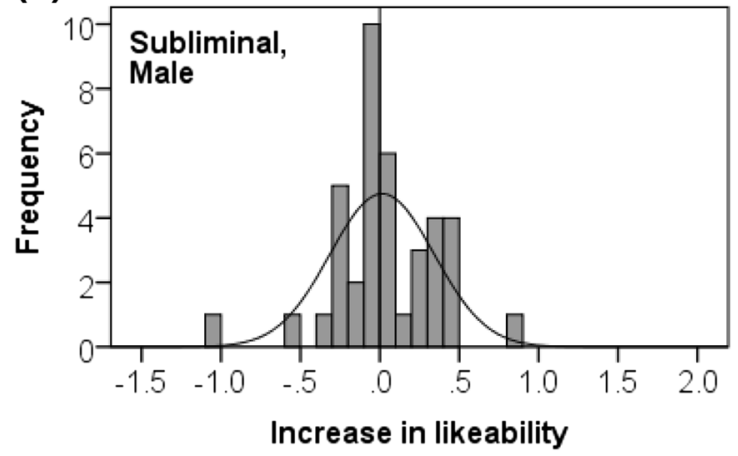

(b)

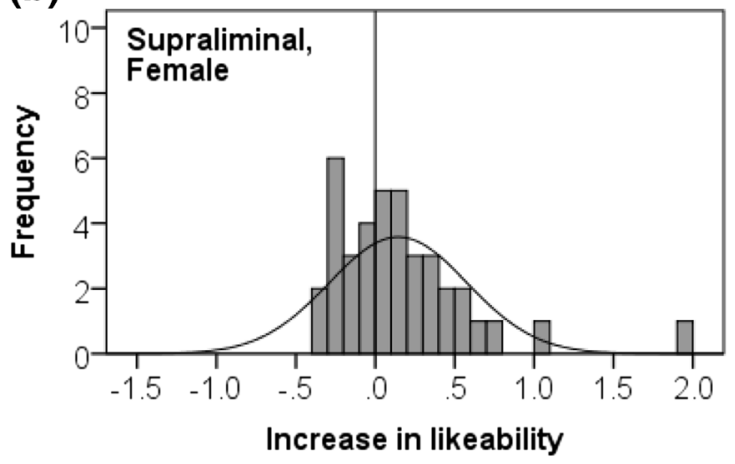

(d)

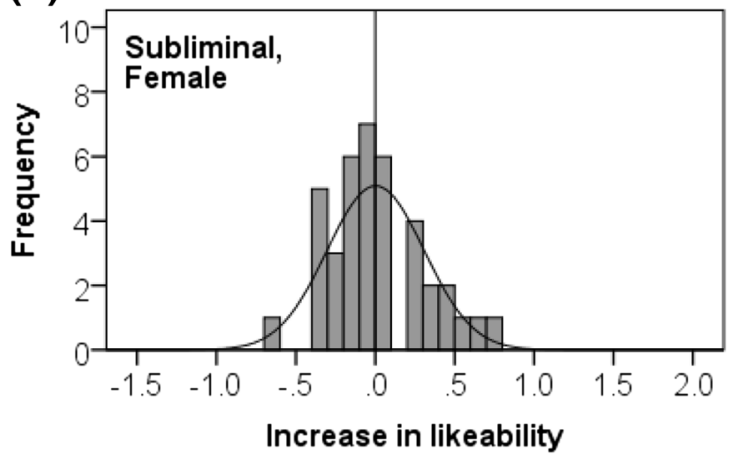

Fig. 4 Histograms showing cueing effects in target evaluations (i.e., average rating of averted targets minus average rating of indicated targets) by gender of the observer and stimulus condition (supraliminal or subliminal). Bell-shaped curves represent the best-fit normal distributions

correlate significantly with that for subliminal arrow markers in either male, $r=.064, p=.70$, or female participants, $r=.26$, $p=.12$.

\section{Experiment 2}

Experiment 1 revealed that the likability of geometric figures was modulated by supraliminal arrow markers, though the effect was enhanced by one participant who showed a strong effect. This result is not consistent with a previous study (Bayliss et al., 2006) that reported a significant increase of likability from gaze cues but not from arrow markers. On the other hand, Tipples and Pecchinenda (2018) recently replicated the Bayliss et al. (2006) experiment using gaze cues among a larger number of participants and reported a gaze cueing effect in object likability that was much smaller than that in the original study. Consequently, we conducted a second experiment to verify the modulation of the likability of geometric figures by both arrow markers and gaze cues.

In Experiment 1, a greater cueing effect was shown for female than for male participants. Therefore, we employed only female participants in this experiment. We also used the empathy quotient (EQ) and the systematic quotient (SQ) to examine individual differences and their relationship with the cueing effect, for both arrow and gaze cues.

\section{Method}

\section{Participants}

Forty female students $\left(M_{\text {age }}=21.9, S D=0.7\right)$ who did not participate in Experiment 1 were recruited from the same Japanese university. We obtained informed consent from all participants, and they received $1,000 ¥$ for their participation. This experiment was approved by the Ritsumeikan University ethics review committee for research involving human participants and was conducted in accordance with the Declaration of Helsinki.

\section{Apparatus}

We used the same display monitor and program used in Experiment 1.

\section{Stimuli}

The arrow markers and nonsensical geometric figures used in Experiment 1 were also used in this experiment. For the experiment with gaze cueing, we employed the ten young East Asian female faces used in our previous study (Mitsuda \& Masaki, 2018). Each consisted of a face looking ahead and two faces looking to the right or left. All faces were created 
using the FaceGen software (Singular Inversions Inc., Toronto, Canada). All of them are attractive and have a smile. The size of the facial images was the same as that of the arrow markers.

\section{Procedure}

Half of the participants carried out a likability evaluation task using arrow markers that was the same as the likeability evaluation task (Task 2) in Experiment 1, followed by the same task using gaze cues. The rest of the participants carried out the tasks in reverse order. In the gaze cue task, the image of the bar was replaced by a face looking ahead, and the images of the arrow markers were replaced by faces looking to the right or left. Each task consisted of 80 tasks. After the recording, the participants answered a Japanese version (Wakabayashi, Baron-Cohen, \& Wheelwright, 2006) of the EQ and SQ questionnaires (Baron-Cohen, 2003). The experiment took about 75 min.

\section{Results}

The likability of the geometric figures pointed to by arrow markers (indicated targets; $M=0.048$ ) was slightly higher than that of those paired with arrows in the opposite direction (averted targets; $M=-0.131$ ), which was congruent with the results of Experiment 1. However, the difference was not statistically significant, $t(39)=1.68, p=.10, d_{z}=0.27$. The analysis, using the combined data of the female participants in Experiments 1 and 2, showed that a significant cueing effect was induced by arrow markers, $t(78)=2.54, p=.013, d_{z}=$ 0.29 .

On the other hand, the likability of geometric figures when gazed at by female faces $(M=0.045)$ was also higher than that of averted targets $(M=-0.179), t(39)=1.73, p=.091, d_{z}=$ 0.27 . However, the effect was not statistically significant. In addition, the effect size was much smaller than that in the study by Bayliss et al. (2006), $d_{z}=0.94$. The effect size observed in the present experiment was almost the same as that reported by Tipples and Pecchinenda (2018), $d_{z}=0.29$.

The cueing effect induced by arrow markers was correlated with that induced by gaze cues $(r=.55, p<.001)$; the magnitude of this effect did not differ significantly depending on whether it was induced by arrow markers $(M=0.18)$ or gaze cues $(M=0.22), t(39)=0.40, p=.70, d_{z}=0.06$.

Figure 5 shows the relationship between the individual traits (EQ and SQ) and the magnitudes of the cueing effects (gaze and arrow) for each participant. EQ did not correlate with the cueing effects induced by either gaze $(r=-.08, p=$ $.62)$ or arrow markers $(r=.18, p=.27)$. However, EQ was correlated with the cueing effect induced by arrow markers when two participants who showed strong cueing effects were eliminated from the data (see Fig. $5 \mathrm{~B}, r=.49, p=.002$ ). SQ did not correlate with the cueing effect induced by arrow markers $(r=.03, p=.88)$. A correlation $(r=.48, p=.002)$ was observed between SQ and the cueing effect induced by gaze. However, the correlation was driven by a number of participants who were strongly affected by the gaze cues, as is shown in Fig. 5C. When five participants whose cueing effects were larger than 1.0 were eliminated from the analysis, the significance disappeared $(r=.18, p=.30)$.

Figure 5 also shows that a number of participants showed strong liking effects induced by both gaze and arrow markers. The two participants who showed a strong effect induced by arrow markers (2.9 and 1.8) also showed strong effects induced by gaze (1.9 and 3.5, respectively). However, we were unable to find any peculiarities of these participants, such as of their relationship with the experimenter. When these two participants were excluded from the analysis, the magnitude of the cueing effect became smaller for both gaze cues (gaze: $M$ $=0.09$, arrow: $M=0.06$ ). In addition, the analysis using the combined data of female participants in Experiments 1 and 2, excluding the participants who showed a strong liking effect induced by arrow markers (i.e., one participant in Exp. 1 and two participants in Exp. 2), showed that the cueing effect was not significant, $t(75)=1.83, p=.071, d_{z}=0.21$. Accordingly, we concluded that the liking effect induced by arrow markers was small, except for a small number of participants who were strongly affected.

\section{Summary and concluding discussion}

In this study we first verified the shifts of spatial attention caused by supraliminal and subliminal arrow markers and compared the shifts by participant gender. As a result, in accordance with previous studies, the reaction times to targets indicated by supraliminal and subliminal arrow markers were shorter than those to targets in the opposite direction. When the supraliminal markers were presented, the cueing effect was greater for female than for male participants. This trend has been observed across different countries (Alwall et al., 2010; Bayliss et al., 2005; Cooney et al., 2017; Deaner et al., 2007; Feng et al., 2011; Liu et al., 2013; Merritt et al., 2007). Therefore, the gender difference in the cueing effect might be due to some biological differences rather than to specific cultural differences. On the other hand, this study showed that, for subliminal arrow markers, there was no significant gender difference in the cueing effect or in the ratio of participants who responded faster to the indicated targets.

Previous studies have shown that the gender difference in the shift of spatial attention differs by the type of visual stimulus (Bayliss et al., 2005; Merritt et al., 2007). Arrow markers cause endogenous attentional shifts, in which observers shift their attention according to the content of the visual stimuli. On the other hand, exogenous attentional shifts, in which 
(a)

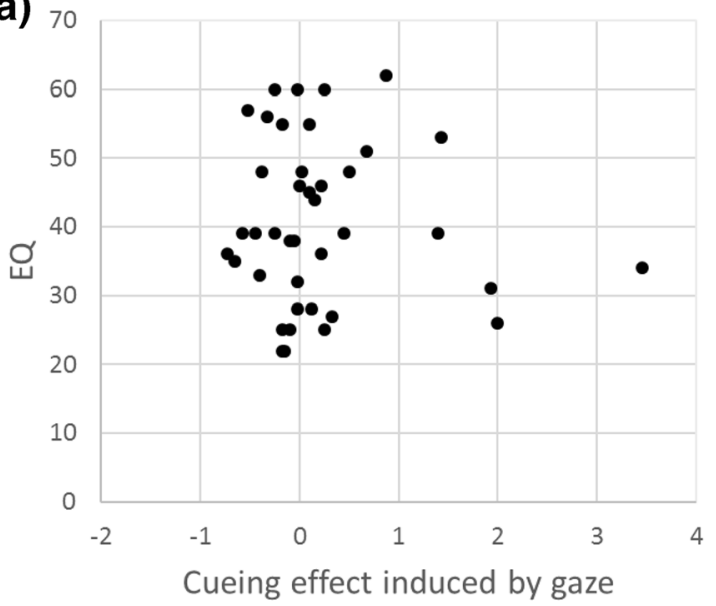

(c)

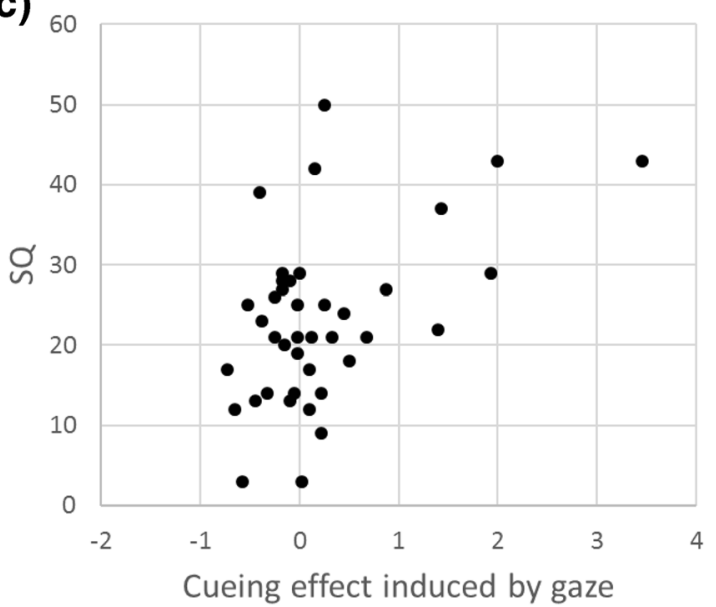

(b)

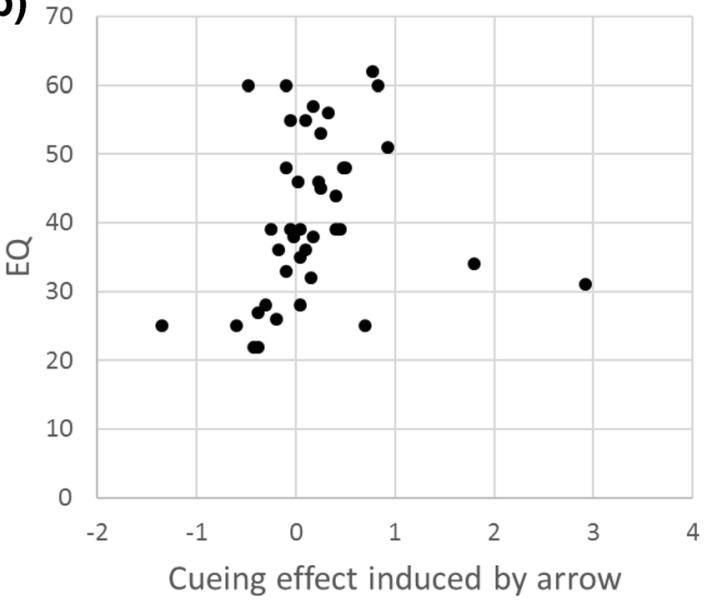

(d)

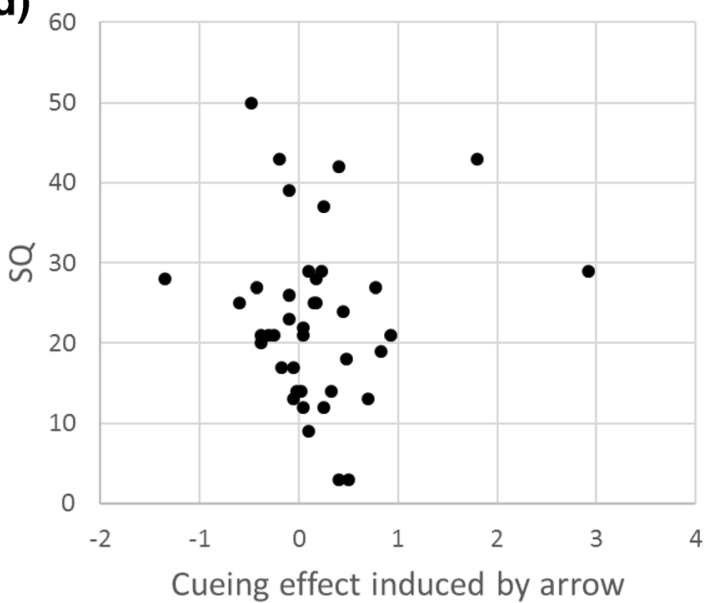

Fig. 5 Scatterplots of individual traits (empathy quotient or systematic quotient: EQ [top row] or SQ [bottom row]) and the cueing effects in object likability induced by gazes (left column) and arrows (right column)

observers shift their attention toward a salient visual stimulus that appears in their peripheral field of view, are caused regardless of the content of the visual stimulus. These two experiments have shown a gender difference for endogenous attentional shifts but not for exogenous attentional shifts. Furthermore, endogenous attentional shift occurs differently according to the gender of observers. Female observers responded more slowly to the target when the arrow markers pointed in the direction opposite that of the target than when no arrow markers appeared. In contrast, male observers responded faster to averted targets than to targets that did not follow any arrow markers (Merritt et al., 2007). These results indicate that the gender difference in endogenous attentional shifts is not a quantitative but a qualitative difference.

One possible factor for the gender difference in the present study was obedience to visual stimuli. Women have higher EQs than men, and people with higher EQs show greater attentional shift under the gaze of other individuals (Alwall et al., 2010). Therefore, female participants might respond more obediently to the arrow markers than male participants do, which might have led to the significant difference between genders found in previous studies and in the present study. On the other hand, finding no significant gender difference for subliminal arrow markers implies that the obedience to symbolic markers affects the observer's behavior only when the markers are perceived consciously.

Previous studies (Gayet et al., 2014; Reuss et al., 2011) have shown that the cueing effect on reaction times to subliminal symbolic markers was significant only when the visual markers were informative (i.e., markers pointed to the targets at a frequency that was higher than chance). However, the present study showed a significant shortening of the reaction time even when the arrow marker direction was determined randomly, which contradicts previous studies. There are several possible explanations for this contradiction.

First, if obedience also modulates the cueing effect for subliminal symbolic markers, individual differences in the participants of each study could have led to these different results. However, in the present study, the cueing effect on reaction times to subliminal arrow markers was not correlated with the effect of supraliminal arrow markers, which does not support the existence of an effect of obedience for subliminal markers. 
A study by Gayet et al. (2014) showed that the shortening of reaction times by noninformative arrow markers was about 10 $\mathrm{ms}$, which was longer than that observed in the present study (6 ms). Therefore, in the present study, a larger number of participants or controls for participants' gender might have increased the significance level of the effect in the noninformative visual-marker condition. Another possible reason for the inconsistency between the present study and previous ones lies in their different ways of presenting visual stimuli. The study by Gayet et al. exploited the phenomenon of binocular rivalry by presenting a cue image to one eye and a mask image to the opposite eye simultaneously, to mask the cue image. In the study by Reuss et al. (2011), a mask image was presented after the image of an arrow marker. In contrast, in the present study we presented an arrow image five times, interspersed among mask images. Therefore, the different methods of presentation might have changed the magnitude of the visual input to the brain or led the obedience effect to produce different results.

Next, in the present study we measured the change in the likability of objects that were indicated by arrow markers. As a result, the likability of the objects pointed to by supraliminal arrow markers was higher than that of objects in the opposite direction, even though the magnitude of the effect was small. One reason for the inconsistency between the present study and that by Bayliss et al. (2006), in which they did not observe a change in likability depending on the direction of supraliminal arrow markers, may be a difference in target stimuli, in addition to differences in participant characteristics, such as culture. Bayliss and colleagues used household items as their target stimuli, whereas in the present study we used nonsensical figures; household items may be more affected by individual preferences than are nonsensical figures. The smaller effect of individual preference in the present study might have enhanced the sensitivity of the study to likability, thereby enabling us to detect a change in likability induced by the arrow markers.

On the other hand, when arrow markers were presented subliminally, there was no significant cueing effect on target evaluations. The results of the target detection task (Task 1) show that subliminal markers did affect observer behavior. Therefore, information on the subliminal markers must be transmitted to the observers' brains without their awareness, thereby shifting their attention in the likability evaluation task (Task 2). Thus, this result is not in accordance with our hypothesis. It implies that the increase in object likability induced by supraliminal arrow markers that we observed in the present study was caused by another higher cognitive function, regardless of the attentional shift. The absence of a significant correlation between the cueing effects in Task 1 (i.e., attentional shift) and 2 (i.e., object likability) also indicates that the attentional shift was unrelated to the increase in object likability.
As for the gender difference in the increase of object likability, in this study we did not detect a statistical significance. In contrast, a significant gender difference was observed in the attentional shift induced by supraliminal markers. This result also did not concur with the hypothesis that an attentional shift increases the preference rating of objects at the location to which the observers attend. On the basis of these results, we concluded that the increase of object likability induced by arrow markers was not due to attentional shift, but rather to a higher cognitive process, such as obedience.

This result also implies that the increase of object likability through subliminal gaze, which was observed in a previous study (Mitsuda \& Masaki, 2018), was also not driven by attentional shift but by a higher cognitive mechanism. As for supraliminal gaze, many previous studies have shown significant gaze cueing effects on object likability and modulation of the effect by the facial properties of the gaze sender, as we described in the introduction. These results also suggest that the increase in object likability induced by gaze cueing is driven by a higher cognitive process. Manera, Elena, Bayliss, and Becchio (2014) showed that gaze cueing caused an attentional shift but did not affect affective evaluations when target objects appeared immediately after the gaze was interrupted by a barrier. In accordance with the present study, their results also showed that attentional shift is not the source of the liking effect induced by gaze cueing.

Recently, Tipples and Pecchinenda (2018) replicated the study by Bayliss et al. (2006) with a larger number of participants and reported a gaze cueing effect in object likability much smaller than that of the original study. Our results also showed that the gaze cueing effect was small and that the magnitude of the effect size was almost same as that observed by Tipples and Pecchinenda. Furthermore, the liking effect induced by gaze cues was not significantly different from that induced by arrow cues. These results were unexpected, because many other studies have shown significant liking effects induced by gaze cues (King et al., 2011; Strick, Holland, \& van Knippenberg, 2008; Treinen, Corneille, \& Luypaert, 2012; Ulloa et al., 2015; van der Weiden et al., 2010).

This study also showed that not all participants, but a small number of them, showed a strong liking effect, which could have been a source of inconsistency between studies. However, the strong cueing effects observed among these participants were not the result of random noise but were driven by individual differences in certain traits. At the same time, in the present study, SQ was still correlated with the liking effect induced by gaze cues, and EQ was correlated with the liking effect induced by arrow cues, when the two participants who showed exceptionally strong effects were excluded. Therefore, individual differences should be given more attention in future studies of the cueing effect.

Assessment of individual differences is also important for studies involving subliminal stimuli. In the present study we 
presented arrow markers with a duration of $8 \mathrm{~ms}$, which was shorter than the durations in previous studies. Most participants were not able to detect the arrow direction; however, one participant was able consciously to detect it correctly, and another participant did not notice it but was nonetheless able to detect the arrow direction at a significantly high rate. In most previous studies, the judgment of a supraliminal or subliminal condition was performed through inquiries and perception tests, as in this study. However, these tests are timeconsuming and are not able to show the magnitude of the visual input to the brain of the observers. In future studies, a method to determine the magnitude of subliminal perception should be developed.

Author note All data and video clips of the trial sequence in this study are available at the Open Science Framework (https://osf.io/f4d3c/).

\section{References}

Alwall, N., Johansson, D., \& Hansen, S. (2010). The gender difference in gaze-cueing: Associations with empathizing and systemizing. Personality and Individual Differences, 49, 729-732. https://oi. org/10.1016/j.paid.2010.06.016

Baron-Cohen, S. (2003). The essential difference. The truth about the male and female brain. New York, NY: Basic Books.

Bayliss, A. P., di Pellegrino, G., \& Tipper, S. P. (2005). Sex differences in eye gaze and symbolic cueing of attention. Quarterly Journal of Experimental Psychology, 58, 631-650. https://doi.org/10.1080/ 02724980443000124

Bayliss, A. P., Frischen, A., Fenske, M. J., \& Tipper, S. P. (2007). Affective evaluations of objects are influenced by observed gaze direction and emotional expression. Cognition, 104, 644-653. https://doi.org/10.1016/j.cognition.2006.07.012

Bayliss, A. P., Paul, M. A., Cannon, P. R., \& Tipper, S. P. (2006). Gaze cuing and affective judgments of objects: I like what you look at. Psychonomic Bulletin \& Review, 13, 1061-1066. https://doi.org/10. 3758/bf03213926

Bayliss, A. P., Schuch, S., \& Tipper, S. P. (2010). Gaze cueing elicited by emotional faces is influenced by affective context. Visual Cognition, 18, 1214-1232. https://doi.org/10.1080/13506285.2010.484657

Becker, M. W. (2010). The effectiveness of a gaze cue depends on the facial expression of emotion: Evidence from simultaneous competing cues. Attention, Perception, \& Psychophysics, 72, 1814-1824. https://doi.org/10.3758/app.72.7.1814

Bornstein, R.F. (1989). Exposure and affect: Overview and meta-analysis of research, 1968-1987. Psychological Bulletin, 106, 265-289. https://doi.org/10.1037/0033-2909.106.2.265

Canadas, E., \& Mast, M. S. (2017). Drawn towards what others seem to like: Implicit preference for objects and people looked at with a Duchenne smile. Motivation and Emotion, 41, 628-635. https:// doi.org/10.1007/s11031-017-9626-6

Cooney, S. M., Brady, N., \& Ryan, K. (2017). Spatial orienting of attention to social cues is modulated by cue type and gender of viewer. Experimental Brain Research, 235, 1481-1490. https://doi.org/10. 1007/s00221-017-4909-4

Deaner, R. O., Shepherd, S. V., \& Platt, M. L. (2007). Familiarity accentuates gaze cuing in women but not men. Biology Letters, 3, 64-67. https://doi.org/10.1098/rsbl.2006.0564
Endo, N., Saiki, J., Nakao, Y., \& Saito, H. (2003). Perceptual judgments of novel contour shapes and hierarchical descriptions of geometrical properties. Japanese Journal of Psychology, 74, 346-353. https:// doi.org/10.4992/jjpsy.74.346

Feng, Q., Zheng, Y. Q., Zhang, X. M., Song, Y., Luo, Y. J., Li, Y. D., \& Talhelm, T. (2011). Gender differences in visual reflexive attention shifting: Evidence from an ERP study. Brain Research, 1401, 5965. https://doi.org/10.1016/j.brainres.2011.05.041

Gabay, S., Avni, D., \& Henik, A. (2012). Reflexive orienting by central arrows: Evidence from the inattentional blindness task. Psychonomic Bulletin \& Review, 19, 625-630. https://doi.org/10. 3758/s13423-012-0252-8

Gayet, S., Van der Stigchel, S., \& Paffen, C. L. E. (2014). Seeing is believing: Utilization of subliminal symbols requires a visible relevant context. Attention, Perception, \& Psychophysics, 76, 489-507. https://doi.org/10.3758/s13414-013-0580-4

Gillath, O., Mikulincer, M., Birnbaum, G. E., \& Shaver, P. R. (2007). Does subliminal exposure to sexual stimuli have the same effects on men and women? Journal of Sex Research, 44, 111-121. https://doi. org/10.1080/00224490701263579

Jones, B. C., DeBruine, L. M., Main, J. C., Little, A. C., Welling, L. L. M., Feinberg, D. R., \& Tiddeman, B. P. (2010). Facial cues of dominance modulate the short-term gaze-cuing effect in human observers. Proceedings of the Royal Society B: Biological Sciences, 277, 617-624. https://doi.org/10.1098/rspb.2009.1575

King, D., Rowe, A., \& Leonards, U. (2011). I trust you; hence, I like the things you look at: Gaze cueing and sender trustworthiness influence object evaluation. Social Cognition, 29, 476-485. https://doi.org/10. 1521/soco.2011.29.4.476

Liu, G., Hu, P. P., Fan, J., \& Wang, K. (2013). Gender differences associated with orienting attentional networks in healthy subjects. Chinese Medical Journal, 126, 2308-2312. https://doi.org/10. 3760/cma.j.issn.0366-6999.20122637

Manera, V., Elena, M. R., Bayliss, A. P., \& Becchio, C. (2014). When seeing is more than looking: Intentional gaze modulates object desirability. Emotion, 14, 824-832. https://doi.org/10.1037/a0036258

Merritt, P., Hirshman, E., Wharton, W., Stangl, B., Devlin, J., \& Lenz, A. (2007). Evidence for gender differences in visual selective attention. Personality and Individual Differences, 43, 597-609. https://doi. org/10.1016/j.paid.2007.01.016

Mitsuda, T., \& Masaki, S. (2018). Subliminal gaze cues increase preference levels for items in the gaze direction. Cognition and Emotion, 32, 1146-1151. https://doi.org/10.1080/02699931.2017.1371002

Niedzwiecka, A., \& Tomalski, P. (2015). Gaze-cueing effect depends on facial expression of emotion in 9-to-12-month-old infants. Frontiers in Psychology, 6, 122:1-11. https://doi.org/10.3389/fpsyg.2015. 00122

Ponari, M., Trojano, L., Grossi, D., \& Conson, M. (2013). “Avoiding or approaching eyes"? Introversion/extraversion affects the gazecueing effect. Cognitive Processing, 14, 293-299. https://doi.org/ 10.1007/s10339-013-0559-z

Posner, M. I. (1980). Orienting of attention. Quarterly Journal of Experimental Psychology, 32, 3-25. https://doi.org/10.1080/ 00335558008248231

Reuss, H., Pohl, C., Kiesel, A., \& Kunde, W. (2011). Follow the sign! Top-down contingent attentional capture of masked arrow cues. Advances in Cognitive Psychology, 7, 82-91. https://doi.org/10. 2478/v10053-008-0091-3

Sato, W., Okada, T., \& Toichi, M. (2007). Attentional shift by gaze is triggered without awareness. Experimental Brain Research, 183, 87-94. https://doi.org/10.1007/s00221-007-1025-x

Serrien, D. J., Sovijärvi-Spapé, M. M., \& Rana, G. (2012). Subliminal priming and effects of hand dominance. Acta Psychologica, 141, 73-77. https://doi.org/10.1016/j.actpsy.2012.07.008

Strick, M., Holland, R. W., \& van Knippenberg, A. (2008). Seductive eyes: Attractiveness and direct gaze increase desire for associated 
objects. Cognition, 106, 1487-1496. https://doi.org/10.1016/j. cognition.2007.05.008

Süßenbach, F., \& Schönbrodt, F. (2014). Not afraid to trust you: Trustworthiness moderates gaze cueing but not in highly anxious participants. Journal of Cognitive Psychology, 26, 670-678. https:// doi.org/10.1080/20445911.2014.945457

Tipples, J., \& Pecchinenda, A. (2018). A closer look at the size of the gaze-liking effect: A preregistered replication. Cognition and Emotion. Advance online publication. https://doi.org/10.1080/ 02699931.2018 .1468732

Treinen, E., Corneille, O., \& Luypaert, G. (2012). L-eye to me: The combined role of need for cognition and facial trustworthiness in mimetic desires. Cognition, 122, 247-251. https://doi.org/10.1016/j. cognition.2011.10.006

Ulloa, J. L., Marchetti, C., Taffou, M., \& George, N. (2015). Only your eyes tell me what you like: Exploring the liking effect induced by other's gaze. Cognition and Emotion, 29, 460-470. https://doi.org/ 10.1080/02699931.2014.919899

van der Weiden, A., Veling, H., \& Aarts, H. (2010). When observing gaze shifts of others enhances object desirability. Emotion, 10, 939-943. https://doi.org/10.1037/a0020501

Wakabayashi, A., Baron-Cohen, S., \& Wheelwright, S. (2006). Individual and gender differences in Empathizing and
Systemizing: Measurement of individual differences by the Empathy Quotient (EQ) and the Systemizing Quotient (SQ). Japanese Journal of Psychology, 77, 271-277. Retrieved from http://search.ebscohost.com/login.aspx?direct=true $\& d b=$ edsbl\&AN=RN196055044\&lang=ja\&site=eds-live

$\mathrm{Xu}$, S., Zhang, S., \& Geng, H. Y. (2011). Gaze-induced joint attention persists under high perceptual load and does not depend on awareness. Vision Research, 51, 2048-2056. https://doi.org/10.1016/j. visres.2011.07.023

Zajonc, R. B. (1968). Attitudinal effects of mere exposure. Journal of Personality and Social Psychology, 9(2, Pt. 2), 1-27. https://doi. org/10.1037/h0025848

Publisher's note Springer Nature remains neutral with regard to jurisdictional claims in published maps and institutional affiliations. 